\title{
Assimetria cerebral e lateralização da linguagem: déficits nucleares na esquizofrenia como indicadores de predisposição genética
}

\author{
Timothy J. Crow*
}

\section{INTRODUÇÃO}

Em 1961, Paul Broca convenceu-se de que Dax ${ }^{1}$, em observações anteriores, estava certo ao afirmar que a faculdade da linguagem se localizava no lado esquerdo do cérebro. Em 1877, ele já considerava a possibilidade de que: "O Homem, entre todos os animais, é aquele que tem o cérebro mais assimétrico. É, também, o que possui a maior quantidade de faculdades adquiridas. Entre essas faculdades... a faculdade da linguagem é a mais nobre. É essa que nos distingue mais nitidamente dos animais"2.

O conceito de que a assimetria é a característica definidora do cérebro humano correu o risco de se perder durante a maior parte do século $X X$. Isso só não ocorreu devido, em grande parte, às teorias genéticas de Annett $^{3}$ e à redescoberta anatômica da

*MD, PhD, FRCPsych, Professor do Departamento de Psiquiatria da Universidade de Oxford (Inglaterra) e Diretor do Prince of Wales International Centre for Research on Schizophrenia and Depression.

Original em inglês publicado em Curr Opin Psychiatry, v.17 - 2004. @ 2004 Lippincott Williams \& Wilkins. Tradução publicada com autorização do autor. assimetria por Geschwind \& Levitsky ${ }^{4}$. A sua relevância para a psicose tornou-se visível através do estudo seminal das psicoses associadas com a epilepsia de lobo temporal de Flor-Henry ${ }^{5}$. Eu argumento que somente através do entendimento do mecanismo de assimetria cerebral os fenômenos da psicose farão algum sentido.

\section{ASSIMETRIAS ANATÔMICAS}

As assimetrias anatômicas do cérebro humano são sutis, e sua base exata na estrutura cortical ainda é pouco conhecida. Talvez a assimetria que mais chame a atenção é aquela do planum temporale, ainda que tenha sido medida de várias maneiras diferentes. Em um estudo post mortem em 21 cérebros adultos, Harasty et al..$^{* *}$ relataram que o neocórtex do planum temporale esquerdo (córtex auditivo secundário) é mais longo e mais fino do que o neocórtex da direita, um resultado que os autores consideraram consistente com um "modelo balão" de desenvolvimento cortical. Em nível microscópico, Buxhoeveden et al. ${ }^{7}$ relataram que as minicolunas formadas pelas 
células piramidais do planum temporale são mais largas e apresentam maior separação do lado esquerdo, e que essas assimetrias distinguem o cérebro humano daquele do chimpanzé e do macaco rhesus.

Esses achados, portanto, comprovam o ponto de vista (o "axioma Broca-Annett") de que a assimetria direcional é a característica que define o cérebro humano. Uma série de estudos recentes testou a hipótese ${ }^{8}$ de que há desvios na assimetria anatômica na esquizofrenia.

\section{Giro temporal superior}

Em um estudo com pacientes esquizofrênicos em primeiro episódio $(n=15)$, MacCarley et al..$^{9^{* *}}$ demonstraram uma reversão da assimetria temporal revertida e menor matéria cinzenta em giro temporal superior posterior (GTS) em relação a pacientes com psicose afetiva $(n=18)$ e controles $(n=18)$; volumes menores de substância cinzenta em planum temporale; volumes de GTS posterior esquerdo e planum temporale esquerdo foram correlacionados com a voltagem potencial evocado P300, que também se constatou ser reduzido no lado esquerdo. Os autores ${ }^{10}$ também observaram que o giro de Heschl esquerdo (o giro anterior ao planum temporale na superfície temporal superior que inclui o córtex auditivo primário) foi $21 \%$ menor no lado esquerdo em 21 homens esquizotípicos versus 22 indivíduos de comparação, como já previamente observado em pacientes esquizofrênicos. O mais interessante foi que, na amostra de pacientes em primeiro episódio submetidos à nova tomografia após 1,5 ano $(\mathrm{n}=$ 13 versus 15 pacientes afetivos em primeiro episódio versus 14 sujeitos normais do grupo de comparação), Kasai et al. ${ }^{1{ }^{* *}}$ relataram uma perda de substância cinzenta de $8,4-9,6 \%$ no GTS esquerdo, não observada no GTS direito ou em qualquer outra região.

Kim et al. ${ }^{12^{*}}$ relataram que, em 25 pacientes do sexo masculino nunca medicados com neurolépticos (comparados a 25 controles), o volume da porção anterior do GTS no lado esquerdo foi inversamente correlacionado os sintomas positivos, enquanto a porção posterior do lado direito apresentou uma correlação positiva com os sintomas negativos. Comparado aos controles, houve uma redução significativa no volume da porção anterior do lado esquerdo em um grupo com sintomas positivos graves e um aumento no volume da porção posterior direita naqueles com sintomas negativos graves, quando comparados aos pacientes com sintomas negativos leves. Os achados sugerem que os detalhes das relações morfológicas do córtex heteromodal perisilviano em ambos os lados são relevantes à forma de psicose.

Collinson et al. ${ }^{13^{*}}$, em um estudo de 33 casos de psicose com início na adolescência comparados com 30 controles normais semelhantes, constataram que a relação entre a assimetria e as manifestações de psicose são sutis e dependem do sexo. Foi observada uma interação significativa entre sexo e diagnóstico na assimetria de volume do hemisfério; mulheres com psicose apresentaram assimetria à direita reduzida em comparação com controles; casos masculinos apresentam assimetria à esquerda reduzida em relação aos controles masculinos. Volume hemisférico esquerdo diminuído nos homens e assimetria hemisférica à direita diminuída em mulheres correlacionaram-se com QI reduzido.

Achados relacionados foram apresentados por Pantelis et al. ${ }^{1 * *}$ ao examinarem imagens seqüenciais de ressonância magnética (RM), com intervalos de pelo menos 12 meses, em 21 sujeitos considerados de alto risco para psicose, 10 dos quais haviam desenvolvido sintomas psicóticos no período interveniente (os outros 11 não). Os pacientes que haviam desenvolvido psicose apresentaram uma redução de substância cinzenta nos córtices parahipocampal, fusiforme e orbitofrontal do lado esquerdo. Embora as mudanças não tenham sido no GTS, como no estudo apresentado por Kasai et al. ${ }^{11^{* *}}$, a lateralização esquerda é de interesse, pois também foi observada em estudos post mortem no $\mathrm{GTS}^{15}$, giros parahipocampais e fusiformes ${ }^{16}$.

Há, portanto, a possibilidade de que esteja ocorrendo uma mudança em áreas do córtex de associação heteromodal, tais como o GTS, envolvido na percepção da fala, e o giro fusiforme, associado com o reconhecimento facial, que penetram no giro hipocampal no hemisfério dominante, isto é, naquele hemisfério responsável pelas representações fonológicas. Se essa conjetura estiver correta, ela justifica as afirmações de Crichton-Browne, em 1879, de que "não parece impossível que aquelas estruturas corticais de evolução mais recente, e que supostamente estão localizadas no lado esquerdo do cérebro, possam ser as primeiras a sofrer na insanidade" 17 .

Embora a lateralidade esquerda do elemento progressivo apontada nos estudos de Kasai et al. ${ }^{11^{* *}}$ e Pantelis et al. ${ }^{14^{* *}}$ seja notável, outro aspecto do estudo elaborado por Pantelis et al. $^{14^{* *}}$ é intrigante. Nas imagens basais da 
amostra total de 75 indivíduos de alto risco, a característica preditora em 23 sujeitos que desenvolveram psicose foi uma redução na substância cinzenta no hipocampo, no giro hipocampal e córtex circundante, no GTS e pólo temporal e no giro frontal inferior direito. Em outras palavras, segundo esse estudo, as alterações preditoras de psicose incipiente e aquelas que realmente ocorrem no decorrer do seu surgimento estão situadas em lados diferentes do cérebro. Dadas as dificuldades técnicas de avaliar as assimetrias anatômicas no cérebro humano, as imagens coletadas nesse estudo e em estudos comparáveis merecem cuidadosa avaliação com o uso de novas técnicas de imagens recentemente desenvolvidas para análise de assimetria (veja a seguir).

Um problema significativo é a relação entre o já conhecido aumento de tamanho ventricular observado na esquizofrenia e as mudanças vistas no córtex. Uma possibilidade é a de que os ventrículos estejam aumentados como resultado de desvios no desenvolvimento sulcogiral em nível do córtex. Em um estudo de 40 pacientes com esquizofrenia e 40 controles usando imagens tridimensionais de alta resolução, Yotsutsuji et al. ${ }^{18^{*}}$ observaram que os aumentos mais significativos nos homens (20 pacientes versus 20 controles) estão localizados no corno temporal esquerdo, com mudanças menos significativas na mesma direção em pacientes do sexo feminino. Esses achados, comparados àqueles de um estudo post mortem anterior ${ }^{19}$, sugerem que alterações ventriculares podem ser um indicador sensível de mudanças o córtex temporal esquerdo. Por exemplo, em um estudo de imagem por RM dos primeiros episódios de psicose, Degreef et al. ${ }^{20}$ encontraram correlatos clínicos de aumento, observados através das medidas do corno temporal esquerdo, mas ausentes em outras regiões do sistema ventricular.

\section{Regiões cinguladas e paracinguladas}

Há particular interesse quanto à região cingulada e paracingulada anterior no aspecto medial do lobo frontal, porque essa região é relacionada a funções comunicativas em primatas e, segundo relatos, portadora de assimetrias em seres humanos. Takahashi et al. ${ }^{21^{*}}$ relataram uma assimetria de volume à direita, tanto na substância cinzenta como na branca, em 20 controles do sexo feminino, com perda dessa assimetria em 20 pacientes do substância cinzenta foi observada nos homens, mas não houve perda em pacientes do sexo masculino. Em 12 pacientes esquizotípicas, foi constatada uma perda comparável de assimetrias de substância cinzenta e branca; o mesmo não ocorreu nos pacientes do sexo masculino 22

Examinando a morfologia (proeminência, presença ou ausência) do sulco paracingulado (que define o giro paracingulado situado na porção externa da curva do giro cingulado), Yucel et al. ${ }^{23^{\star *}}$ relataram uma perda da assimetria normal à esquerda em 55 pacientes esquizofrênicos do sexo masculino, em comparação com 75 controles normais do sexo masculino. Os autores ${ }^{24^{*}}$ encontraram uma ausência similar de assimetria em 63 homens com "risco ultra-elevado" de psicose, mas não encontraram qualquer diferença entre os 21 que desenvolveram psicose posteriormente e os que não desenvolveram quando comparados ao mesmo grupo controle. Com um método similar para avaliar a presença e a integridade do sulco paracingulado, Le-Provost et al..$^{25^{*}}$ encontraram uma perda de assimetria em 40 pacientes destros do sexo masculino com esquizofrenia estabelecida pelo Diagnostic and Statistical Manual of Mental Disorders (DSM), versão IV, em comparação com 100 controles destros.

A significância desses achados na região cingulada/paracingulada é que aqui foram estabelecidas assimetrias confiáveis não encontradas facilmente em outras áreas dos lobos frontais. No estudo elaborado por Pantelis et al. ${ }^{14^{* *}}$ que, presumivelmente, incluiu alguns dos indivíduos de alto risco estudados por Yucel et al. ${ }^{24^{*}}$, a região cingulada anterior, bilateralmente, foi identificada tanto como uma região que definia aqueles indivíduos com risco de transição para psicose, quanto como uma região que apresentava alteração naqueles que realmente desenvolveram psicose. Há uma indicação clara da necessidade de outros estudos mais aprofundados da estrutura detalhada dessa região, inclusive a lateralização das alterações.

Outra assimetria nos lobos frontais foi relatada por Zhou et al. ${ }^{26}$ que, em um estudo de $R M$, encontraram em 53 pacientes com esquizofrenia comparados com 48 controles pareados por idade e sexo, uma redução no volume de substância branca no ramo anterior da cápsula interna e um aumento na assimetria direita maior que a esquerda. A relação entre essa assimetria, até então pouco estudada, e as outras assimetrias aqui revisadas não é clara. Igualmente, uma assimetria de volume à 
direita do pulvinar foi detectada no cérebro post mortem (e também observada em imagens de $\mathrm{RM})$, mas é significativamente reduzida em pacientes com esquizofrenia ${ }^{27^{*}}$.

\section{Imagem do tensor de difusão}

A possibilidade de que a trajetória de trechos de substância branca possa ser definida pela técnica de imagem do tensor de difusão (ITD), que se utiliza das restrições ao movimento de prótons impostas pela topografia do axônio, gerou entusiasmo considerável. Kubicki et al. ${ }^{28^{* *}}$ compararam 15 pacientes com esquizofrenia definida pelo DSM-IV com 18 controles pareados por idade e sexo. Os autores utilizaram imagens do tensor de difusão, com varredura em linhas de $4 \mathrm{~mm}$ de espessura, para gerar mapas fracionais de anisotropia da região do fascículo uncinado ligando os córtices frontal e temporal. Relataram que as medidas da área de seção transversal e anisotropia fracional no ponto mais denso e sobre a área definida como o fascículo uncinado indicavam assimetria (esquerda maior do que a direita) nos sujeitos de comparação, fato ausente em pacientes com uma interação "Grupo - porlado" significativa.

Em um estudo similar que comparou 30 pacientes que preencheram os critérios de esquizofrenia do DSM-IV com 30 controles, Burns et al. ${ }^{29^{*}}$ encontraram reduções em medidas fracionais de anisotropia para regiões que definiram como correspondentes aos fascículos uncinado e arqueado no lado esquerdo, mas ausentes no lado direito do cérebro. Embora os autores tenham afirmado que não testaram especificamente a hipótese de assimetria (de que a alteração primária na esquizofrenia está na relação entre estruturas em ambos os lados do cérebro) através da investigação de uma interação "lado e por diagnóstico", observaram que os seus achados são consistentes com essa hipótese.

Em contraste com os achados desses dois estudos há aqueles de um estudo post mortem $^{30^{*}}$ no qual a área, densidade de fibra e número de fibras do fascículo uncinado foram avaliados por métodos estereológicos no ponto de entrada no lobo temporal em 17 pacientes com esquizofrenia e em 21 controles. Os dois principais achados foram: presença de uma assimetria; o fascículo uncinado com $27 \%$ mais fibras e $33 \%$ maior na área do lado direito do que do lado esquerdo do cérebro. Nenhuma mudança nessa assimetria estava presente nos pacientes.

A discrepância entre os achados dos dois estudos de ITD e o estudo post mortem é de importância considerável. As técnicas são diferentes, mas tanto Highley et al. ${ }^{30^{*}}$ como Kubicki et al. ${ }^{38^{* *}}$ procuraram avaliar o fascículo no ponto onde ele entra no tronco temporal, embora o plano da secção seja diferente entre os estudos, já que é coronal no estudo de ITD e atravessa o tronco temporal no estudo post

Figura 1 - Assimetria do processo de captação de dopamina em indivíduos normais e pacientes com esquizofrenia (reproduzido com permissão de Hsiao et al. ${ }^{31^{* *}}$ )

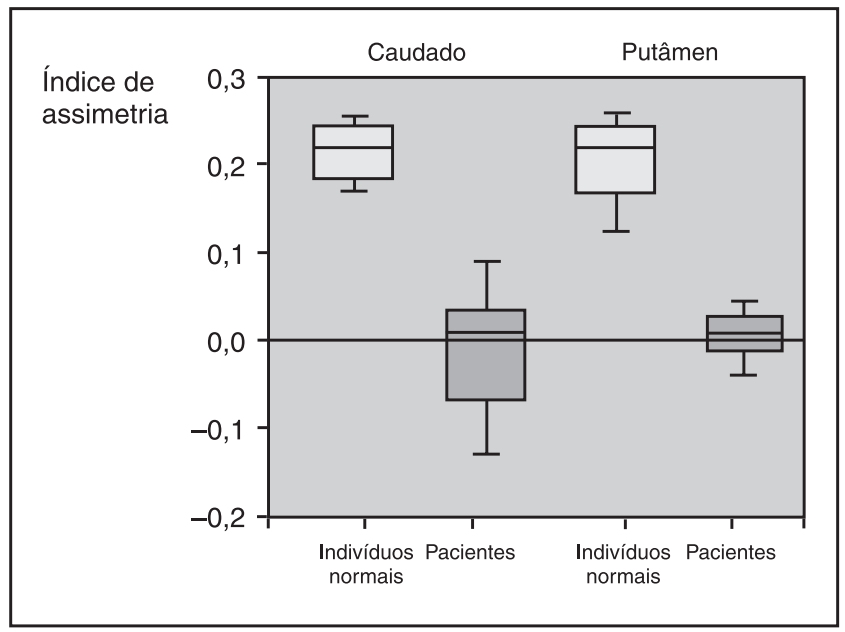


mortem. A assimetria (da direita para esquerda) no estudo por Highley et al..$^{30^{*}}$ foi maior do que na direção oposta àquela detectada por Kubicki

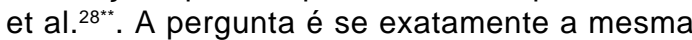
estrutura foi avaliada ou se há componentes críticos da via fibrosa que foram incluídos em um estudo, mas não no outro. Tais questões deveriam ser abordadas por uma nova análise dos dados de RM existentes, com o uso de técnicas delineadas para a identificação de assimetrias.

\section{Outras anomalias da assimetria anatômica}

Uma série de achados relacionados a perdas ou anomalias de assimetria tem sido observada no curso de outras investigações. Hsiao et al. ${ }^{31^{* *}}$, usando a técnica de tomografia por emissão de fóton único, investigaram a captação de dopamina em 12 pacientes com esquizofrenia nunca medicados e em 12 controles. Os autores não encontraram uma mudança global na captação, mas observaram que a assimetria direita-esquerda da fixação no caudado e putâmen, detectada nos controles, havia desaparecido no grupo de pacientes $(p=$ 0,002 ). Os autores compararam a perda de assimetria observada com perdas semelhantes de assimetria relatadas em estudos de captação de fluorodopa, desenvolvido por Hietala et al. ${ }^{32}$, e de atividade de transporte de dopamina, de Laakso et al. ${ }^{33}$.

Uma justificativa surpreendente para o papel da assimetria na patogênese da psicose foi encontrada por Hennessy et al.$^{34}$ através de uma reconstrução da estrutura craniofacial utilizando distâncias entre pontos (interlandmark). Em 169 pacientes com esquizofrenia por (DSM-III-R), comparados com 78 controles normais pareados, os autores constataram que, em ambos os sexos, a altura facial inferior era alongada, e a superior, encurtada; a face era mais larga, e o násio, localizado mais posteriormente. Controles masculinos apresentaram uma assimetria direcional acentuada, enquanto os pacientes do sexo masculino apresentaram assimetria menor ( $p<0,01)$; os controles do sexo feminino apresentaram pouca assimetria, mas, em pacientes, foi observada assimetria acentuada $(p<0,001)$. Os autores concluíram que há uma disrupção sutil da trajetória tridimensional de crescimento craniofacial embrionário-fetal, com uma disrupção da assimetria normal relacionada ao sexo. Os achados são similares àqueles encontrados em estudos post mortem sobre a estrutura cerebral ${ }^{15,16,35,36}$, embora ainda seja necessária uma descrição precisa da trajetória do desenvolvimento de assimetria nos dois sexos.

\section{ASSIMETRIAS FUNCIONAIS}

A pesquisa sobre a atividade neuronal foi centrada em dois focos: o potencial de imagem de RM funcional, particularmente em relação à linguagem, e um enfoque no paradigma específico de mismatch negativity (negatividade de descombinação) como correlato putativo do processo de doença.

\section{Ressonância magnética funcional}

Sommer et al. ${ }^{37^{*}}$ examinaram a dominância hemisférica para linguagem em um protocolo que incluiu tarefas de geração de verbos e decisão semântica e derivaram um índice de lateralidade da ativação dos dois lados em áreas de linguagem frontal, temporal e temporoparietal. Em 12 pacientes do sexo masculino com esquizofrenia, o valor do índice foi de 0,35, comparado com 0,63 em 12 controles, com evidências de ativação aumentada no hemisfério direito e relação inversa entre o índice e a gravidade das alucinações. Em um estudo paralelo envolvendo 12 pacientes do sexo feminino e 12 controles, Sommer et al. ${ }^{38^{*}}$ derivaram índices de 0,44 para as pacientes e 0,75 para as controles, com evidências de um aumento similar na ativação do lado direito no grupo de pacientes. Ao compararem os dois estudos, os autores concluíram que os achados não diferiam nos dois sexos.

Aplicando o mesmo protocolo a 12 pares de gêmeos monozigóticos normais e 12 pares discordantes para esquizofrenia, Sommer et al. ${ }^{39}$ demonstraram índice de lateralização reduzido nos pares discordantes, mas não distinguiram os indivíduos doentes dos normais. Concluíram que a lateralização de linguagem diminuída constitui uma predisposição genética para a esquizofrenia.

Bleuler ${ }^{40}$ considerou que transtorno de pensamento estava no cerne da esquizofrenia, e há embasamento para esse ponto de vista em estudos de estrutura da linguagem ${ }^{41^{*}}$. Kircher et al. ${ }^{42,43^{* *}}$ selecionaram seis pacientes para a presença de transtorno da forma do pensamento. Os pacientes foram submetidos ao teste de Rorschach durante 3 minutos, enquanto eram avaliados por $R M$, em comparação a seis controles. Os autores constataram que: (1) a gravidade do transtorno 
de pensamento tinha uma correlação negativa com a ativação nos giros temporais superior esquerdo e médio e uma correlação positiva com a ativação do caudado direito e giro precentral e verme do cerebelo esquerdo ${ }^{42}$; (2) nos controles, a quantidade de fala está correlacionada com a ativação do GTS esquerdo, enquanto, nos pacientes, correlaciona-se com a ativação à direita ${ }^{43^{* *}}$.

Esses achados, portanto, oferecem um suporte sólido para o conceito de que há uma lateralização anormal da linguagem na esquizofrenia, pelo menos em pacientes com transtorno do pensamento.

Em outro componente dessa série de investigações ${ }^{44}$, os mesmos pacientes e controles foram comparados com um grupo de seis pacientes sem transtorno da forma do pensamento em testes de completar frases, tanto com uma palavra gerada por eles próprios (geração) quanto com uma escolha entre duas palavras alternativas (decisão), ou com leitura. Pacientes com transtorno do pensamento desordenado apresentavam menos ativação do córtex temporal direito quando a geração de palavras foi comparada com a decisão e a leitura, independentemente de outros aspectos do desempenho. Esses pacientes, que ativavam o GTS direito em vez do esquerdo em relação à taxa de produção de palavras nos seus solilóquios de Rorschach, ativaram o córtex temporal direito com menos freqüência do que qualquer um dos dois grupos controle quando solicitados a realizar a tarefa específica de completar uma frase do seu próprio repertório de palavras. Kircher et al. ${ }^{44}$ implicaram o córtex temporal direito na compreensão da linguagem no nível de discurso e sugeriram que este é o nível no qual se manifesta o transtorno de pensamento.

Essa é uma possibilidade. Outro contexto dentro do qual esses achados podem ser considerados é pressupor que os engramas fonológicos, motores ou sensoriais são normalmente lateralizados para o hemisfério dominante, e, conseqüentemente, as associações ou "significados", em particular aqueles mais remotos, isto é, menos freqüentes, são parcialmente lateralizados para 0 hemisfério não-dominante. Na esquizofrenia, parece que essa segregação é menos seguramente estabelecida. O que é testado no paradigma de Kircher et al. ${ }^{42}$ é o acesso aos engramas sensoriais armazenados no decorrer da recuperação de palavras, seja no discurso sem restrições em um solilóquio de Rorschach ou para completar uma frase com um fim significativo. No fluxo livre no solilóquio de Rorschach, indivíduos normais acessam engramas em regiões perisilvianas (giros superior temporal e supramarginal) no lado esquerdo, isto é, próximo às suas representações fonológicas. Eles estão fazendo algumas associações remotas. Ao contrário, os indivíduos com transtorno do pensamento fazem as suas associações de Rorschach sobretudo nas regiões correspondentes do lado direito, isto é, longe das suas representações fonológicas primárias, mas onde, aparentemente, estão as associações sensóriomotoras mais prontas. Por outro lado, na tarefa de completar frases, os indivíduos são obrigados a acessar significados que não estão imediatamente associados com o engrama fonológico (a frase não é uma fórmula decorada). Indivíduos normais executam essa tarefa ativando as suas associações mais remotas, isto é, aquelas localizadas nas regiões perisilvianas do lado direito. Indivíduos com transtornos de pensamento, com diferenciação hemisférica menos bem estabelecida entre associações remotas e freqüentes, têm menor capacidade de execução. Nessas circunstâncias, esses indivíduos apresentam menor ativação do que os controles e os pacientes sem transtorno do pensamento do GTS no lado direito.

Esses achados são relacionados principalmente com aspectos sensoriais da linguagem e com os lobos posteriores do cérebro. Walter et al. ${ }^{45^{* *}}$ utilizaram RM funcional para investigar a função de lobo frontal em uma tarefa de memória de trabalho verbal e espacial em 15 pacientes com esquizofrenia agudamente doentes e 15 sujeitos controle pareados por idade. Não houve evidência da hipofrontalidade previamente relatada, mas, enquanto o grupo controle apresentou uma dominância de memória de trabalho verbal no lobo frontal inferior no lado esquerdo e dominância da memória espacial no córtex prefrontal direito, esses efeitos de dominância estavam ausentes nos pacientes, isto é, não houve lateralização. Lawrie et al. ${ }^{46}$ investigaram oito pacientes com esquizofrenia segundo o DSM-IV e os compararam com 10 controles usando RM funcional e tarefa de completar frases. Encontraram significativamente menos correlações entre o córtex prefrontal temporal esquerdo e dorsolateral esquerdo em pacientes e correlações negativas com alucinações auditivas. Os autores argumentam a respeito de uma redução da conectividade frontotemporal, mas a lateralidade do achado é importante. 
Um estudo de potenciais evocados desenvolvido por Gonzalez-Hernandez et al. ${ }^{47}$ lançou nova luz sobre as investigações da função do lobo frontal e da conectividade. Os autores examinaram a relação entre 0 desempenho no Wisconsin Card Sort Test com amplitudes de potenciais evocados em locais homólogos bilaterais ao longo do eixo ânteroposterior. Quando comparados a 10 indivíduos normais, 12 pacientes com esquizofrenia apresentaram uma perda de assimetria esquerda na banda teta, observada em controles nas regiões posterior anterior e média, e uma assimetria anômala à esquerda, atravessando as bandas teta, alfa e beta2, circunscrita às regiões occipitais. Como esses potenciais anormais surgiram cedo no processamento de informações e precederam os potenciais anteriores, os autores concluíram que as anomalias occipitais são relevantes para as anormalidades da função do lobo frontal, já amplamente relatadas em outros estudos.

Em um estudo incomum de imagem por tomografia de emissão de pósitrons, Heckers et al. ${ }^{48^{*}}$ investigaram o fluxo sangüíneo em uma tarefa de visualização passiva com duas exposições sucessivas a um padrão de ruído visual estacionário. Enquanto que os sujeitos controle apresentaram, na segunda exposição, uma diminuição no fluxo sangüíneo no hemisfério direito, os pacientes apresentaram um aumento, acompanhado por uma diminuição à esquerda. Esses achados contribuem para as evidências que surgem, sugerindo que aspectos de lateralização funcional são perdidos ou revertidos na psicose.

\section{Mismatch negativity}

A mismatch negativity (anomalias da resposta eletrofisiológica a um estímulo inesperado) foi sugerida por alguns como a chave da fisiopatologia da esquizofrenia. Não há unanimidade de que os aspectos críticos das anomalias sejam lateralizados. Usando magnetoencefalografia, Pekkonen et al. ${ }^{49}$ examinaram a mismatch negativity em 15 pacientes esquizofrênicos e 19 controles e constataram uma redução em ambos os hemisférios e uma demora na resposta, que foi lateralizada à esquerda. Com um desenho similar, mas combinando magnetoencefalografia com um protocolo de RM funcional em 12 pacientes e 12 controles, Kircher et al. ${ }^{50}$ encontraram uma perda de amplitude usando magnetoencefalografia em ambos os hemisférios em pacientes, mas um efeito de duração lateralizado à direita apenas em controles, isto é, a assimetria foi perdida no grupo de pacientes. Com RM funcional, a assimetria de duração foi localizada no planum temporale, ou seja, no córtex de associação auditiva, e novamente a assimetria foi perdida nos pacientes.

Usando registros eletroencefalográficos em 15 pacientes esquizofrênicos, Youn et al. ${ }^{51^{*}}$ observaram uma assimetria de potência na resposta da mismatch negativity à esquerda nos controles e à direita nos pacientes $(p<$ $0,001)$, com uma perda do coeficiente de assimetria à esquerda correlacionada com sintomas positivos, especialmente alucinações, e sintomas negativos.

Como extensão de uma série importante de investigações magnetoencefalográficas, Rojas et al. ${ }^{52^{*}}$ examinaram potenciais evocados de campo auditivo em 19 pacientes com esquizofrenia e 22 controles. A relação esperada entre profundidade e freqüência fo encontrada no grupo de comparação, mas não nos pacientes $(p<0,004)$. Além disso, a assimetria normal ântero-posterior do potencial N100 foi reduzida nas cinco freqüências de estímulo. Se, conforme sugerem os autores, a organização tonotópica do córtex auditivo é perturbada na esquizofrenia, isso tem implicações na interpretação dos achados de estudos com estímulos auditivos, tais como aqueles envolvidos no paradigma da mismatch negativity, e a perda de assimetria anatômica é critica.

Um estudo recente de $\mathrm{RM}$ funcional ${ }^{53}$ esclarece significativamente o papel de estímulos relacionados à fala no paradigma do tipo descombinação (mismatch). Ngan et al..$^{53}$ apresentaram estímulos relacionados e não relacionados à fala a 14 pacientes com esquizofrenia e 29 sujeitos normais em um delineamento aleatório (odd-ball) - isto é, com a apresentação de um estímulo inesperado segundo a abordagem da mismatch negativity e calcularam a diferença entre os estímulos em termos da resposta do fluxo sangüíneo. Comparados aos controles, os indivíduos com esquizofrenia apresentaram maior ativação diferencial a estímulos da fala versus não-fala no córtex temporal direito, córtex frontal superior esquerdo e junção temporoparietal esquerda. A magnitude da diferença na região da junção temporoparietal esquerda foi correlacionada com a gravidade do pensamento desorganizado. Os autores concluíram que o "estudo suporta a hipótese de que a lateralização funcional aberrante da fala é uma característica subjacente a esquizofrenia". Fica claro que nenhum 
progresso pode ser esperado de paradigmas de descombinação, a não ser que a lateralização e a relação de fala versus não-fala seja considerada.

\section{GENÉTICA DA PSICOSE}

Em uma revisão da literatura sobre a lateralização cerebral (19 estudos de destreza manual, 10 de escuta dicótica e 39 de assimetria anatômica) em esquizofrenia, Sommer et al. ${ }^{54}$ resumiram sua meta-análise indicando que: (1) a prevalência de não-destros foi significativamente mais elevada em pacientes esquizofrênicos quando comparados com controles saudáveis e psiquiátricos; (2) estudos de escuta dicótica limitados àqueles envolvendo tarefas de consoantes/vogais e palavras fusionadas demonstraram evidências de lateralização aumentada; e (3) houve diminuição significativa das assimetrias do planum temporale e da fissura silviana. Os autores observaram fortes evidências de lateralização cerebral diminuída na esquizofrenia e que "encontrar o gene de dominância cerebral poderia decifrar a predisposição genética para a esquizofrenia" (um estudo anterior ${ }^{55}$ examinou assimetrias morfológicas em famílias multiplamente afetadas e concluiu que havia um fator genético envolvido).

A potencial importância dessa conclusão é aumentada pelo aparente fracasso da abordagem de ligação (linkaje) na identificação de genes para psicose. Duas meta-análises de estudos de ligação na esquizofrenia e na doença bipolar não demonstraram achados consistentes. A importância da questão é enfatizada pela conclusão de que a esquizofrenia é uma condição específica dos seres humanos ("o preço que o Homo sapiens paga pela linguagem") - para um panorama geral dessa teoria, ver Berlim et al. ${ }^{56^{*}}$ - e que a assimetria cerebral é a característica que define a espécie (o axioma de Broca-Annett).

Onde está localizado e o que é o gene para dominância cerebral? Com base nos prejuízos associados a aneuploidias dos cromossomos sexuais, sugeri ${ }^{57}$ que o gene para a assimetria está localizado em uma região de homologia entre os cromossomos $\mathrm{X}$ e $\mathrm{Y}$. Tornou-se claro que uma região de homologia entre a região Xq21.3 e dois blocos no braço curto do cromossomo $\mathrm{Y}$ foi estabelecida no decorrer da evolução hominídea por uma transposição duplicativa, e que parte dessa região foi submetida a subseqüente inversão paracêntrica. Seqüências de genes dentro desse bloco estão, portanto, presentes tanto no cromossomo $Y$ como no cromossomo $X$ em seres humanos, mas apenas no $X$ em outros primatas e, até onde se sabe, em outros mamíferos. Dentro desse bloco, foi identificado um par de genes (Protocaderina $X$ e $Y$ ) que tem estado sob seleção positiva de domínio (Williams N \& Crow TJ, em preparação) e que codifica para moléculas de adesão da superfície celular, expressas no cérebro, inclusive na camada de células germinais do córtex. Esse par de genes é, portanto, um candidato a determinante de características cerebrais, incluindo a assimetria, que distinguem os seres humanos dos outros primatas. $O$ fato de que a seqüência de genes no cromossomo $Y$ difere daquela do cromossomo $X$ sugere que essa diferença é relevante para as diferenças sexuais no que se refere à taxa de crescimento cerebral (mais rápida), destreza (mais forte) e aquisição de palavras (mais precoce no sexo feminino), relevantes ao desenvolvimento da linguagem e que ainda precisam ser explicados.

Será que as variações no par de genes da Protocaderina $X / Y$ explicam a genética da assimetria e da psicose? A concordância do mesmo sexo, marca da transmissão de um gene homólogo $X-Y$, foi relatada no que se refere à destreza manual ${ }^{58}$, esquizofrenia e doença maníaco-depressiva ${ }^{59}$. Evidências de ligação tanto à psicose quanto à destreza manual foram buscadas no cromossomo X. Apesar de alguns achados equivocados em relação à destreza manual, por exemplo, na qual foi observado um pico modesto em relação à região Xq21.3 da homologia $^{60}$, os achados em relação à psicose foram negativos ${ }^{61,62}$. Todavia, dado o fracasso geral da abordagem de ligação, toda a questão da natureza da predisposição genética requer reavaliação. Será possível que a predisposição não esteja relacionada à variação de seqüência, isto é, que seja de uma natureza invisível à estratégia de ligação?

Essa possibilidade certamente se origina no caso de um par de genes como Protocaderina $X$ e $Y$, no qual o homólogo no $Y$ surgiu na história evolucionária recente. Genes homólogos X-Y são protegidos da inativação de $\mathrm{X}$, embora o mecanismo através do qual isso ocorre (possivelmente como resultado de pareamento $X-Y$ em meiose masculina) seja obscuro no momento. É concebível que a inativação ou imprinting de $\mathrm{X}$ ou alguma combinação desses dois processos explique a predisposição genética?

Os achados dos estudos com gêmeos são relevantes. Se a discordância entre gêmeos 
chegar a $50 \%$ e não tiver sido identificada qualquer diferença ambiental entre a experiência dos gêmeos que explique a discordância ${ }^{63}$, isso em si sugere que fatores epigenéticos (talvez expressos como diferenças na trajetória do desenvolvimento cerebral) desempenham um papel. Há indicações de que a variação epigenética determina a variação na dominância cerebral e na assimetria. Em um estudo significativo, Steinmetz et al. ${ }^{64}$ constataram que, em pares de gêmeos monozigóticos discordantes para destreza manual, a assimetria do planum temporale era menor no gêmeo canhoto, isto é, a assimetria anatômica estava relacionada à assimetria funcional, e essa correlação não podia ser explicada por uma seqüência de DNA compartilhada. $\mathrm{Na}$ série de gêmeos monozigóticos discordantes para esquizofrenia ${ }^{65,66}$ do National Institute of Mental Health, há diferenças na assimetria anatômica entre os gêmeos, tanto no volume da substância cinzenta no lobo temporal como no comprimento da fissura silviana. $\mathrm{O}$ gêmeo doente apresentou um volume menor de substância cinzenta no lobo temporal posterior do lado esquerdo e menor assimetria do segmento posterior da fissura silviana ${ }^{67}$.

$\mathrm{Na}$ RM funcional de gêmeos monozigóticos discordantes para esquizofrenia realizada por Sommer et al. ${ }^{39}$, a lateralização para linguagem não separou os gêmeos doentes dos normais, mas em um relato de caso de um único par discordante ${ }^{68}$, usando um paradigma de fluência verbal, foi observada uma ativação de RM mais disseminada e funcional bilateral no gêmeo doente em oposição à uma ativação mais restrita, limitada ao lado esquerdo, no gêmeo normal. Outros estudos de lateralização da linguagem com diferentes RM funcionais e paradigmas eletrofisiológicos são claramente indicados (veja comentário de Picchioni69).

Temos, assim, evidências de que: (1) a dimensão (assimetria) que define a espécie reflete uma variação que é epigenética; (2) a variação que predispõe à psicose é relacionada à assimetria e é epigenética; e (3) o par de genes Protocaderina $X / Y$, que é o principal candidato a determinante da assimetria (o torque), está em situação evolucionária nova no que se refere à sua expressão epigenética.

Parece que esses fatos devem estar relacionados e que um entendimento genético da psicose requer a elucidação do mecanismo de proteção da inativação do cromossomo $X$ de genes que estão representados em forma homóloga no cromossomo $\mathrm{Y}^{70^{*}}$. Um relato de
Klinefelter ( $X X Y)$ que desenvolveu psicose e aparentemente diferia de outros indivíduos com síndrome de Klinefelter nos índices de assimetria anatômica à RM ilustra a possível relevância de tais genes.

\section{MÉTODOS DE ESTUDO DA ASSIMETRIA CEREBRAL}

A aplicação da morfometria baseada em voxel à avaliação da assimetria cerebral foi descrita por Good et al. ${ }^{72}$ e Watkins et al. ${ }^{73}$. Métodos de análise de assimetria foram revisados por Thompson \& Toga ${ }^{74^{*}}$. Uma nova técnica de quantificação do torque cerebral por $\mathrm{RM}$ foi relatada ${ }^{75^{*}}$ e aplicada a uma população clínica. Uma questão não resolvida é por que a perda de assimetria na esquizofrenia, descrita com uma técnica de região de interesse em cortes coronais por Bilder et al. ${ }^{76}$, não é mais prontamente detectada mediante o método quantitativo. Esse contraste levanta a questão relativa a se o torque reflete uma mudança na estrutura sulcogiral em vez de uma mudança no volume das áreas relevantes do córtex em ambos os lados e se é possível pressupor que a linha média seja uma estrutura planar. O mesmo grupo $^{77}$ relatou que, em 37 homens em primeiro episódio de esquizofrenia comparados com 27 controles, houve uma reversão significativa das assimetrias de volume observadas no cerebelo anterior e posterior; nos controles, a assimetria de lobo anterior estava à direita e, nos pacientes, localizava-se à esquerda, enquanto que a assimetria do lobo posterior estava à esquerda em controles e à direita em pacientes. Há aqui a possibilidade de que as assimetrias no cerebelo reflitam o torque no córtex cerebral. O fato de que anomalias sejam observadas em homens mas não em mulheres com esquizofrenia e sejam revertidas ao longo do eixo ântero-posterior é similar aos achados de um estudo anterior sobre medidas de comprimento através da superfície dorsal do córtex ${ }^{35}$, pelo fato de haver uma interação com o sexo ao longo do eixo ânteroposterior. Pode ser que o cerebelo abra uma nova perspectiva para a solução do problema. A definição do planum temporale foi investigada por Meisenzahl et al. ${ }^{78}$, que, ao contrário do resultado da meta-análise de Sommer et al. ${ }^{54}$, não encontraram uma anomalia de assimetria em 30 pacientes do sexo masculino com esquizofrenia, comparados com 30 controles do sexo masculino. Os motivos dessa discrepância são de extremo interesse. Uma possibilidade é que a mudança seja mais da forma do que do volume, conforme sugerido por Harasty et al. ${ }^{6^{* *}}$. 


\section{CONCLUSÃO}

A assimetria cerebral (o torque do occipital frontal direito ao occipital esquerdo) é a característica que define o cérebro humano e, conforme sugeriu Broca, é a base neural da linguagem. Se a esquizofrenia é uma condição específica do ser humano (o preço pago pelo Homo sapiens pela linguagem), ela somente pode ser entendida através do torque. Nenhuma outra dimensão pode fazer com que os achados dos estudos anatômicos, funcionais e genéticos tenham sentido. As evidências dos estudos de imagem anatômicos e post mortem revisadas aqui demonstram que há um grau menor de lateralização. Em diversos desses estudos, incluindo os de estrutura craniofacial, há uma interação entre a anomalia da assimetria e o sexo. Os estudos funcionais reforçam o conceito de que a lateralização da linguagem é reduzida e sugerem que os componentes da linguagem, normalmente confinados ao hemisfério esquerdo, estão ou localizados mais bilateralmente, ou realmente foram revertidos, isto é, estão localizados no hemisfério direito. Estudos em gêmeos monozigóticos discordantes acrescentam evidências cruciais de que a variação subjacente à psicose é relacionada à assimetria anatômica e é de caráter "epigenético", isto é, não se relaciona à seqüência de genes, mas à sua expressão. Identificou-se que um par de genes (Protocaderinas $X$ e $Y$ ) foi criado por uma duplicação no decorrer da evolução hominídea e submetido à pressão seletiva positiva, isto é, a seleção para mudança. É expresso como uma molécula de adesão aderência à superfície celular no cérebro, inclusive na camada de células germinais do córtex cerebral. Pode explicar uma diferença de sexo, sendo candidato à assimetria cerebral e a ser portadora da variação epigenética relacionada à psicose.

\section{REFERÊNCIAS E LEITURAS RECOMENDADAS}

Trabalhos de especial interesse, publicados dentro do período anual de revisão, foram destacados como: *de especial interesse; ${ }^{* *}$ de interesse destacado.

1. Dax M. Lésions de la moitié gauche de l'éncephale coincident avec l'oublides signes de la pensée (Read at congrés méridional at Montpelier in 1836). Gaz Hebdom Med Chirurg 1865;11:259-60.

2. Broca P. Rapport sur un memoire de M. Armand de Fleury intitulé: de l'inegalité dynamique des deux hemisphères ceré braux. Bull Acad Med 1877;6:508-39.
3. Annett M. Handedness and brain asymmetry: the right shift theory. Hove, Sussex: Psychology Press; 2002.

4. Geschwind N, Levitsky W. Human brain: left-right asymmetry in temporal speech region. Science 1968;161:186-7.

5. Flor-Henry P. Psychosis and temporal lobe epilepsy, a controlled investigation. Epilepsia 1969;10:363-95.

6. Harasty J, Chan P, Halliday G, et al. The left human speech-processing cortex is thinner but longer than the right. Laterality 2003;8:247-60.

7. Buxhoeveden D, Switala AE, Litaker M, et al. Lateralization of minicolumns in human planum temporale is absent in nonhuman primate cortex. Brain Behav Evol 2001;57:349-58.

8. Crow TJ. Temporal lobe asymmetries as the key to the etiology of schizophrenia. Schizophr Bull 1990;16:43343.

9. McCarley RW, Dean MD, Salisbury DF, et al. Association between smaller left posterior superior temporal gyrus volume on magnetic resonance imaging and smaller left temporal P300 amplitude in first-episode schizophrenia. Arch Gen Psychiatry 2002;59:321-31.

10. Dickey CC, McCarley RW, Voglmaier MM, et al. Smaller left Heschl's gyrus volume in patients with schizotypal personality disorder. Am J Psychiatry 2002;159:1521-7.

11. Kasai K, Shenton ME, Salisbury DF, et al. Progressive decrease of left superior temporal gyrus gray matter volume in patients with first-episode schizophrenia. Am J Psychiatry 2003;160:156-64.

12. Kim JJ, Crespo-Facorro B, Andreasen NC, et al. Morphology of the lateral superior temporal gyrus in neuroleptic naive patients with schizophrenia: relationship with symptoms [abstract]. Schizophr Res 2003;60:181.

13. Collinson SL, Mackay CE, James AC, et al. Brain volume, asymmetry and intellectual impairment in relation to sex in early-onset schizophrenia. $\mathrm{Br} J$ Psychiatry 2003;183:114-20

14. Pantelis C, Velakoulis D, McGorry PD, et al. Neuroanatomical abnormalities before and after onset of psychosis: a cross-sectional and longitudinal MRI comparison. Lancet 2003;361:281-8.

15. Highley JR, McDonald B, Walker MA, et al. Schizophrenia and temporal lobe asymmetry. A post-mortem stereological study of tissue volume. $\mathrm{Br} \mathrm{J}$ Psychiatry 1999;175:127-34

16. McDonald B, Highley JR, Walker MA, et al. Anomalous asymmetry of fusiform and parahippocampal gyrus grey matter in schizophrenia: a post-mortem study. Am J Psychiatry 2000;157:40-7.

17. Crichton-Browne J. On the weight of the brain and its component parts in the insane. Brain 1879;2:42-67.

18. Yotsutsuji T, Saitoh O, Suzuki M, et al. Quantification of lateral ventricular subdivisions in schizophrenia by highresolution three-dimensional magnetic resonance imaging. Psychiatry Res Neuroimag 2003;122:1-12.

19. Crow TJ, Ball J, Bloom SR, et al. Schizophrenia as an anomaly of development of cerebral asymmetry. A postmortem study and a proposal concerning the genetic basis of the disease. Arch Gen Psychiatry 1989;46:114550.

20. Degreef G, Ashtari M, Bogerts B, et al. Volumes of ventricular system subdivisions measured from magnetic resonance images in first episode schizophrenic patients. Arch Gen Psychiatry 1992;49:531-7.

21. Takahashi T, Kawasaki $\mathrm{Y}$, Kurokawa K, et al. Lack of normal structural asymmetry of the anterior cingulate gyrus in female patients with schizophrenia: a volumetric magnetic resonance imaging study. Schizophr Res 2002;55:69-81.

22. Takahashi T, Suzuki M, Kawasaki, et al. Volumetric magnetic resonance imaging study of the anterior 
cingulate gyrus in schizotypal disorder. Eur Arch Psychiatry Clin Neurosci 2002;252:268-77.

23. Yucel M, Stuart GW, Maruff $P$, et al. Paracingulate morphological differences in males with established schizophrenia: a magnetic resonance imaging morphometric study. Biol Psychiatry 2002;52:15-23.

24. Yucel M, Wood SJ, Phillips LJ, et al. Morphology of the anterior cingulate cortex in young men at ultra-high risk of developing a psychotic illness. Br J Psychiatry 2003;182:518-24.

25. Le-Provost J-B, Bartrés-Faz D, Palliere-Martinot M-L, et al. Paracingulate morphology in men with early onset schizophrenia. Br J Psychiatry 2003;182:228-32.

26. Zhou S-Y, Suzuki M, Hagino H, et al. Decreased volume and increased asymmetry of the anterior limb of the internal capsule in patients with schizophrenia. Bio Psychiatry 2003;54:427-36.

27. Highley JR, Walker MA, Crow TJ, et al. Low medial and right lateral pulvinar volumes in schizophrenia: a postmortem study. Am J Psychiatry 2002;160:1177-9.

28. Kubicki M, Westin CF, Maier SE, et al. Uncinate fasciculus findings in schizophrenia: a magnetic resonance diffusion tensor imaging study. Am J Psychiatry 2002;159:813-20.

29. Burns J, Job D, Bastin ME, et al. Structural disconnectivity in schizophrenia: a diffusion tensor magnetic resonance imaging study. Br J Psychiatry 2003;182:439-43.

30. Highley JR, Walker MA, Esiri MM, et al. Asymmetry of the uncinate fasciculus: a post-mortem study of normal subjects and patients with schizophrenia. Cerebr Cort 2002;12:1218-224.

31. Hsiao M-C, Lin K-J, Liu C-Y, et al. Dopamine transporter change in drug-naive schizophrenia: an imaging study with $99 \mathrm{mTc}$-TRODAT-1 patients. Schizophr Res 2003;65:39-46.

32. Hiet ala J, Syvalahti E, Vilkman H, et al. Depressive symptoms and presynaptic dopamine function in neuroleptic naive schizophrenia. Schizophr Res 1999;35:41-50.

33. Laakso A, Vilkman $\mathrm{H}$, Alakare B, et al. Striatal dopamine transporter binding in neuroleptic-naive patients with schizophrenia studies with positron emission tomography. Am J Psychiatry 2000;157:269-71.

34. Hennessy RJ, Lane A, Kinsella A, et al. 3D reconstruction and morphometric analysis of cranial dysmorphology and gender-related asymmetries in schizophrenia as an index of cerebral dysmorphogenesis. Schizophr Res. In press 2004.

35. Highley JR, Esiri MM, Cortina-Borja M, et al. Anomalies of cerebral asymmetry in schizophrenia interact with gender and age of onset: a post-mortem study. Schizophr Res 1998;34:13-25.

36. Highley JR, Esiri MM, McDonald B, et al. The size and fibre composition of the corpus callosum with respect to gender and schizophrenia: a post-mortem study. Brain 1999;122:99-110.

37. Sommer IEC, Ramsey NE, Kahn RS. Language lateralization in schizophrenia, an fMRI study. Schizophr Res 2001;52:57-67.

38. Sommer IEC, Ramsey NE, Mandl RWC, et al. Language lateralization in women with schizophrenia. Schizophr Res 2003;60:183-90.

39. Sommer IEC, Ramsey NE, Mandl RWC, et al. Language activation in monozygotic twins discordant for schizophrenia. Br J Psychiatry. In press 2004.

40. Bleuler E. Dementia Praecox or the group of schizophrenias. [Translated by J Zinkin]. New York: International Universites Press; 1950.

41. Ceccherini-Nelli A, Crow TJ. Disintegration of the components of language as the path to a revision of Bleuler's and Schneider's concepts of schizophrenia. Br J Psychiatry 2003;182:233-40.
42. Kircher TTJ, Liddle PF, Brammer MJ, et al. Neural correlates of formal thought disorder in schizophrenia. Arch Gen Psychiatry 2001;58:769-74.

43. Kircher TTJ, Liddle PF, Brammer MJ, et al. Reversed lateralization of temporal activation during speech production in thought disordered patients with schizophrenia. Psychol Med 2002;32:439-49.

44. Kircher TTJ, Bullmore ET, Brammer MJ, et al. Differential activation of temporal cortex during sentence completion in schizophrenic patients with and without formal thought disorder. Schizophr Res 2001;50:27-40.

45. Walter $\mathrm{H}$, Wunderlich AP, Blankenhorn M, et al. No hypofrontality, but absence of prefrontal lateralization comparing verbal and spatial working memory in schizophrenia. Schizophr Res 2003;61:175-84.

46. Lawrie SM, Buechel C, Whalley HC, et al. Reduced fronto-temporal functional connectivity in schizophrenia associated with auditory hallucinations. Biol Psychiatry 2002;51:1008-11.

47. Gonzalez-Hernandez JA, Pita-Alcorta C, Cedeno I, et al. Abnormal functional asymmetry in occipital areas may prevent frontotemporal regions from achieving functional laterality during WCST performance in patients with schizophrenia. Schizophr Res 2003;61:229-33.

48. Heckers S, Goff D, Weiss AP. Reversed hemispheric asymmetry during simple visual perception in schizophrenia. Psych Res Neuroimag 2002;116:25-32.

49. Pekkonen $\mathrm{E}$, Katila $\mathrm{H}$, Ahveninen $\mathrm{J}$, et al. Impaired temporal lobe processing of preattentive auditory discrimination in schizophrenia. Schizophr Bull 2002;28:467-74.

50. Kircher TTJ, Rapp A, Grodd W, et al. Mismatch responses in schizophrenia: a combined fMRI and whole-head MEG study. Am J Psychiatry. In press 2004.

51. Youn T, Park H-J, Kim J-J, et al. Altered hemispheric asymmetry and positive symptoms in schizophrenia: equivalent current dipole of auditory mismatch negativity. Schizophr Res 2002;59:253-60.

52. Rojas DC, Bawn SD, Carlson JP, et al. Alterations in tonotopy and auditory cerebral asymmetry in schizophrenia. Biol Psychiatry 2002;52:32-9.

53. Ngan ETC, Vouloumonos A, Cairo TA, et al. Abnormal processing of speech during oddball target detection in schizophrenia. Neuroimage 2003;20:889-97.

54. Sommer IEC, Aleman A, Ramsey NE, et al. Handedness, language lateralization and anatomical asymmetry in schizophrenia: meta-analysis. Brit J Psychiatry 2001;178:344-51.

55. DeLisi LE, Sakuma M, Kushner M, et al. Anomalous cerebral asymmetry and language processing in schizophrenia. Schizophr Bull 1997;23:255-71.

56. Berlim MT, Mattevi BS, Blemonte-de-Abreu P, et al. The etiology of schizophrenia and the origin of language: overview of a theory. Compr Psychiatry 2003;44:7-14.

57. Crow TJ. The case for an $X-Y$ homologous determinant of cerebral asymmetry. Cytogenet Cell Genet 1994;67:3934.

58. Corballis MC, Lee K, McManus IC, et al. Location of the handedness gene on the $X$ and $Y$ chromosomes. Am J Med Genet (Neuropsychiatr Genet ) 1996;67:50-2.

59. Crow TJ. Con: the demise of the Kraepelinian binary system as a prelude to genetic advance. In: Gershon ES, Cloninger CR, eds. Genetic approaches to mental disorders. Washington: American Psychiatric Press; 1994. p. 163-92.

60. Laval SH, Dann J, Butler RJ, et al. Evidence for linkage to psychosis and cerebral asymmetry (relative hand skill) on the X chromosome. Am J Med Genet (Neuropsychiatr Genet) 1998;81:420-7.

61. DeLisi LE, Shaw S, Sherrington $R$, et al. Failure to 
replicate evidence for linkage on the $\mathrm{X}$ chromosome in 301 families with schizophrenia or schizoaffective disorder. Am J Med Genet (Neuropsychiatr Genet) 2000;96:335-41.

62. Nicholson T, Yang J, DeLisi LE, et al. Allele sharing for schizophrenia and schizo-affective disorder within a region of Homo sapiens specific XY homology. Am J Med Genet 2002;114:637-40

63. Torrey EF, Bowler AE, Taylor EH, et al. Schizophrenia and manic-depressive disorder. New York: Basic Books; 1994.

64. Steinmetz H, Herzog A, Schlaug G, et al. Brain (a)symmetry in monozygotic twins. Cereb Cort 1995;5:296-300.

65. Suddath RL, Christison GW, Torrey EF, et al. Anatomical abnormalities in the brains of monozygotic twins discordant for schizophrenia. N Engl J Med 1990;322:789-94.

66. Bartley AJ, Jones DW, Torrey EF, et al. Sylvian fissure asymmetries in monozygotic twins: a test of laterality in schizophrenia. Biol Psychiatry 1993;34:853-63.

67. Crow TJ. Commentary on Klaening: twin studies of psychosis and the genetics of cerebral asymmetry. $\mathrm{Br} \mathrm{J}$ Psychiatry 1999;175:399-401.

68. Spaniel F, Hájek T, Tintra J, et al. Differences in fMRI and MRS in a monozygotic twin pair discordant for schizophrenia (case report). Acta Psychiatr Scand 2003;107:155-8.

69. Picchioni M. Invited comment. Acta Psychiatr Neurol Scand 2003;107:155-7.

70. Crow TJ. Handedness, language lateralisation and anatomical asymmetry: relevance of protocadherin $X Y$ to hominid speciation and the aetiology of psychosis. $\mathrm{Br} \mathrm{J}$ Psychiatry 2002;181:295-7.

71. Warwick MM, Lawrie SM, Beveridge A, et al. Abnormal cerebral asymmetry and schizophrenia in a subject with Klinefelter's syndrome $(X X X Y)$. Biol Psychiatry 2003;53:627-9

72. Good CD, Johnsrude I, Ashburner J, et al. Cerebral asymmetry and the effects of sex and handedness on brain structure: a voxel-based morphometric analysis of 465 normal adult human brains. Neuroimage 2001; $14: 685-700$

73. Watkins KE, Paus T, Lerch JP, et al. Structural asymmetries in the human brain: a voxel-based statistical analysis of $142 \mathrm{MRI}$ scans. Cereb Cort 2001;11:868-77.

74. Toga AW, Thompson PM. Mapping brain asymmetry. Nat Rev Neurosci 2003;4:37-48.

75. Mackay CE, Barrick TR, Roberts N, et al. Application of a new image analysis technique to study brain asymmetry in schizophrenia. Psychiatry Res Neuroimag 2003;124:25-35.

76. Bilder RM, Wu H, Bogerts $B$, et al. Absence of regional hemispheric volume asymmetries in first episode schizophrenia. Am J Psychiatry 1994;151:1437-47.

77. Szeszko PR, Gunning-Dixon F, Ashtari M, et al. Reversed cerebellar asymmetry in men with first episode schizophrenia. Biol Psychiatry 2003;53:450-9

78. Meisenzahl EM, Zetzsche T, Preuss U, et al. Does the definition of the borders of the planum temporale influence the results in schizophrenia? Am J Psychiatry 2002;159:1198-200.

\section{RESUMO}

Objetivo: Assimetria cerebral (o torque do frontal direito em relação ao occipital esquerdo) é a característica definidora do cérebro humano e, conforme proposto por Broca, o correlato neural putativo da linguagem. Se, conforme sugerido, a esquizofrenia é o preço que Homo sapiens precisa pagar pela linguagem, o torque, juntamente com seus correlatos funcionais, é de fundamental importância. São revisadas recentes evidências obtidas a partir de estudos anatômicos, funcionais e genéticos.

Achados recentes: Estudos de imagem, post mortem e anatômicos demonstram evidências de uma redução ou reversão de aspectos de assimetria, particularmente no córtex de associação occipitotemporoparietal. Em alguns estudos, há interação com o sexo. Há evidências de que uma alteração no lobo temporal esquerdo é, às vezes, progressiva. Estudos funcionais acrescentam credibilidade ao conceito de que a lateralização da linguagem é reduzida e, em alguns casos, revertida.

Resumo: A dimensão da assimetria se destaca como a variável que pode dar significância às observações entre campos de investigação e que proporciona uma solução para a base genética da psicose. Estudos de gêmeos monozigóticos discordantes têm apresentado fortes indicações de que a variação relevante é epigenética; isso é consistente com a possibilidade de que a variação seja relacionada a alterações estruturais recentes (a transposição duplicativa Xq21.3/Yp) dos cromossomos sexuais.

Descritores: Assimetria cerebral, linguagem, psicose, sapiens, esquizofrenia.

\section{ABSTRACT}

Purpose of review: Cerebral asymmetry (the torque from right frontal to left occipital) is the defining feature of the human brain, and as Broca proposed, the putative neural correlate of language. If as has been suggested schizophrenia is the price that Homo sapiens pays for language, the torque together with its functional correlates is of central significance. Recent evidence from anatomical, functional and genetic studies is reviewed.

Recent findings: Both post-mortem and anatomical imaging studies show evidence of a reduction or reversal of aspects of asymmetry particularly in the occipito-temporo-parietal association cortex. In some studies there is an interaction with sex. There is evidence that change in the left temporal lobe is sometimes progressive. Functional studies add substance to the concept that the lateralization of language is reduced and in some aspects reversed.

Summary: The dimension of asymmetry stands out as the variable that can make sense of observations across fields of investigation, and provides a key to the genetic basis of psychosis. Discordant monozygotic twin studies have indicated strongly that the relevant variation is epigenetic; this is consistent with the possibility that the 
variation is related to recent structural changes (the $X q 21.3 / Y p$ duplicative transposition) on the sex chromosomes.

Keywords: Cerebral asymmetry, language, psychosis, sapiens, schizophrenia.

Title: Cerebral asymmetry and the lateralization of language: core deficits in schizophrenia as pointers to the genetic predisposition

\section{RESUMEN}

Objetivo: Asimetría cerebral (el torque del frontal derecho al occipital izquierdo) es la característica definidora del cerebro humano $y$, conforme al propuesto por Broca, el correlato neuronal putativo del lenguaje. Si, conforme a lo sugerido, la esquizofrenia es el precio que el Homo sapiens debe pagar por el lenguaje, el torque, juntamente con sus correlatos funcionales, es de importancia fundamental. Se revisan recientes evidencias obtenidas a partir de estudios anatómicos, funcionales y genéticos.

Hallazgos recientes: Estudios de imagen, post mortem y anatómicos, demuestran evidencias de una reducción o reversión de aspectos de asimetría, particularmente en la corteza de asociación occipitotemporo-parietal. En algunos estudios, hay interacción con el sexo. Hay evidencias de que una alteración en el lóbulo temporal izquierdo es, a veces, progresiva. Estudios funcionales agregan credibilidad al concepto de que la lateralización del lenguaje es reducida $y$, en algunos casos, revertida.

Resumen: La dimensión de la asimetría se destaca como la variable que puede dar significación a las observaciones entre campos de investigación y que proporciona una solución para la base genética de la psicosis. Estudios de gemelos monocigóticos discordantes vienen presentando fuertes indicaciones de que la variación relevante es epigenética; ello es consistente con la posibilidad de que la variación esté relacionada a alteraciones estructurales recientes (la trasposición replicativa $X q 21.3 / Y p)$ de los cromosomas sexuales.

Palabras clave: Asimetría cerebral, lenguaje, psicosis, sapiens, esquizofrenia.

Título: Asimetría cerebral y lateralización del lenguaje: déficits nucleares en la esquizofrenia como indicadores de predisposición genética

Endereço para correspondência:

Timothy J. Crow

SANE POWIC, Warneford Hospital, Oxford

OX3 7JX, United Kingdom

Fone: (+44) 01865-455917

Fax: (+44) 01865-455922

E-mail: tim.crow@psych.ox.ac.uk

Copyright (C) Revista de Psiquiatria do Rio Grande do Sul - SPRS 